\title{
A large scale morphodynamic process-based model of the Gironde estuary
}

\author{
C. Villaret $^{1}$, N. Huybrechts ${ }^{1,2}$ and A.G. Davies ${ }^{3}$ \\ ${ }^{1}$ Saint Venant Laboratory for Hydraulics (Université Paris Est, joint research unit EDF R\&D - CETMEF - Ecole des Ponts \\ ParisTech), 6 quai Watier, 78400 Chatou, France, catherine.villaret@edf.fr \\ ${ }^{2}$ Roberval Laboratory, LHN (joint research unit UTC-CETMEF), UMR CNRS 6253, Centre de recherches de Royallieu, B.P. \\ 20529, 60206 Compiègne Cedex, France \\ ${ }^{3}$ School of Ocean Sciences, Bangor UniversityMenai Bridge, Anglesey LL59 5AB, UK
}

\begin{abstract}
We present here our effort to develop a morphodynamic model of the Gironde estuary, using a process-based approach. In this complex and strongly dynamical environment, internal coupling between the flow (Telemac-2d) and sediment transport models (Sisyphe) allows the representation of detailed sediment processes and interactions with the bed, using a bed roughness feedback method. In this complex and highly heterogeneous environment, the sediment bed composition had to be schematized assuming 3 classes of bed material. Model results could be further improved by accounting for the cohesive sediment (consolidation processes). Thanks to parallelization of the codes, the simulation takes only $18 \mathrm{hrs}$ on 8 processors (linux station) to calculate the 5-year bed evolution, at the basin scale $(150 \mathrm{~km})$.
\end{abstract}

\section{INTRODUCTION}

Estuaries are located worldwide at the boundary between the marine and terrestrial spheres. They represent the most dynamic sedimentary environment, as well as a highly valuable area for the diversity of their ecosystems. They evolve in response to tidal (marine), wind and wave forcing (atmospheric) and fluvial influence. This highly energetic environment is also characterized by its high non-homogeneity in sediment types and bed features, with mixtures of marine sand input at the mouth of the estuary and muddy fine sediment entering from the rivers. The richness of the flora and fauna adapts locally to the diversity of the morphodynamic features: tidal marshes are a nesting area for migrating birds, flood channels bring in marine fish, while ebb channels influence nutrients and fresh water species in the system.

Most estuaries and bays located on the French Atlantic shore have endured strong alluvial bed evolutions in the last decades. These bed evolutions affect the morphodynamic equilibrium, with important consequences for various economic activities and environmental issues. The diversity of the ecosystems can be endangered under the pressure of economic development (industries, tourism, and navigation). The effect of human interference needs to be monitored in order to preserve the environmental equilibrium and ensure sustainable development. Numerous applications include the impact of dredging activities, construction of dikes, etc... on the morphodynamic equilibrium and their consequences on the biodiversity.

Morphodynamic processes in estuaries are driven by complex interactions between various hydrodynamic, biological and morphological processes. The morphodynamic response acts at a wide range of time scales, which may vary from hours to days (short term), from months to decades (medium term), hundreds of years (long term) or millennia (geological scales). In term of spatial scales, bedforms range from micro-scale bed features (ripples, megaripples of the order of $10 \mathrm{~cm}$ to meters, dunes, sand banks; ebb and flood channels can be classified as meso-scale (100 $\mathrm{m}$ to $\mathrm{km})$, whereas the entire estuary evolves at the macroscale $(10-100 \mathrm{~km})$. Different approaches can be applied to model the morphodynamic response of the estuarine system, depending on the scale of application. Geo-morphodynamic models, also called behavior-oriented models, are designed for long term simulation. They can be applied for example to study the effect of global changes (e.g. sea level rise) on the large scale morphodynamic features. They are based on empirical rules and expert analysis of long term bathymetric data. This approach is however limited by the lack of physical insight [e.g. Karunarathna et al., 2008]. Process based models are designed to represent the detailed physical interaction between the flow and the sediment transport processes. The filtering scale is the time step of the model (of the order of a few seconds) which allows representation with great accuracy of the detailed mean flow variation for a particular combination of tidal signal, atmospheric and flooding event [e.g. Huybrechts, et al., 2011]. Spatial variations in the bathymetry are filtered at the model grid size which varies from $10-100 \mathrm{~m}$, such that the small scale bed features need to be parameterized by bed roughness coefficients.

Despite their complexity, process based models still rely on some empirical parameterization of the complex flow/sediment transport interactions. They are mainly designed to study the short term morphodynamic response of the system to human interference [Chini and Villaret, 2007].The objective of this paper is to discuss the state of the art and the limits of process models to study the medium to long term bed evolutions in complex estuarine systems. Morphodynamic factors have been extensively applied in order to reduce the computational cost [van der Wegen and Roelvink, 2008]. Model predictions could be extended over geological time scales, leading to realistic as well as stable model results [van der Wegen, 2010]. However, this simplifying method introduces an additional source of uncertainty and becomes 
questionable in the presence of mixed sediment, where consolidation and biological processes interact at their own time scales. Thanks to the optimization of numerical schemes, parallelism, as well as tremendous progress in the performance of computers, bed evolution can be calculated on basin scale (10-100 $\mathrm{km}$ ) and for the medium term (years to decades), without the use of hydrodynamic filtering methods.

Our framework is the open source finite element Telemac system (http//www.opentelemac.com) which has been developed over 20 years at EDF R\&D. Our case study is the Gironde macrotidal estuary located South West of France, which is the largest estuary in Western Europe. The tide propagates approximately $170 \mathrm{~km}$ inland from the Bay of Biscay. Its importance to the economy is related to the presence of several ports, including the harbor of Bordeaux as well as various industries, fisheries and tourism activities. Despite the large density of population and its important economic development, the Gironde estuary remains one of the most natural estuaries [Natura, 2000].

We start in Part II with a brief review of existing morphodynamic process-based models and present in more detail the open source Telemac system. In Part III, we discuss some of the local/short term and global issues which can be addressed by a two-dimensional process morphodynamic model. In Part IV, we present the large scale (2D depth-averaged) morphodynamic model of the Gironde estuary.

\section{MORPHODYNAMIC MODELS}

After a short review of existing process-based morphodynamic models, we present here the finite element Telemac system (focusing on 2D modules) which is used in the large scale morphodynamic application.

\section{Review of morphodynamic models}

Numerous morphodynamic models have been developed and applied in the past 30 years in order to predict the sediment transport rates and resulting bed evolution in complex environments. Comprehensive morphodynamic modeling systems like ECOMSed [Hydroqual, 2002], Mike21 [Warren and Bach, 1992], Delft3D [Lesser et al., 2004] and ROMS [Warner et al., 2008] generally include different flow options (from 1D to 3D), a wave propagation model and a sand transport model including bed-load and suspended load (for a review, see for example Papanicolaou et al. [2008]).

Most existing morphodynamic modeling systems rely on finite difference methods and are therefore constrained by the use of boundary fitted (orthogonal curvilinear horizontal coordinate systems, sigma stretched vertical coordinates) which are only suitable for simplified geometry.

\section{The Telemac system}

Like other comprehensive models, the Telemac system comprises various modules to calculate the flow (Telemac-2d or $3 \mathrm{~d}$ ), the waves (Tomawac) and the sediment transport (Sisyphe), which can be chained or internally coupled [cf. Villaret et al., 2011]. In comparison with other comprehensive modeling systems, the main originality lies in the efficiency and flexibility of the finite elements. All modules of the Telemac system are based on unstructured grids and finite-element or finite volume algorithms. The sources and user manuals can be downloaded from the Telemac website: http://www.telemacsystem.com. One important feature is parallelism with domain decomposition.

Different numerical methods are available, as described by Hervouet [2007]. The method of characteristics, kinetic schemes and others can be applied to calculate the convective terms in the momentum equation. The wave equation as well as providing a method of smoothing free surface instabilities is particularly well suited for large scale applications. The use of implicit schemes enables relaxation of the CFL limitation on time steps (typically, values of Courant numbers up to 10 or 50 are acceptable).

For the treatment of tidal flats, a new algorithm based on segments ensures positive water depths and mass conservation without extra limitation of the time step [Hervouet et al., 2010].

The Telemac-2D flow module solves the shallow water equations (momentum and continuity), with several options for the horizontal diffusion terms (depth-averaged k- $\varepsilon$, Elder model (1959) or constant eddy viscosity models) and source terms (atmospheric pressure gradients, Coriolis force ...).

$$
\left.\begin{array}{c}
\frac{\partial h}{\partial t}+\frac{\partial h U}{\partial x}+\frac{\partial h V}{\partial y}=0 \\
\left.\frac{\partial U}{\partial t}+U \frac{\partial U}{\partial x}+V \frac{\partial U}{\partial y}=-g \frac{\partial Z_{s}}{\partial x}+\frac{\tau_{x}}{h}+\frac{1}{h} \operatorname{div}(h v \operatorname{grad}(U))\right) \\
\frac{\partial V}{\partial t}+U \frac{\partial V}{\partial x}+V \frac{\partial V}{\partial y}=-g \frac{\partial Z_{S}}{\partial y}+\frac{\tau_{y}}{h}+\frac{1}{h} \operatorname{div}(h v \operatorname{grad}(V))
\end{array}\right\}
$$

Where $\mathrm{h}$ is the water depth, and $\mathrm{U}, \mathrm{V}$ the horizontal mean velocity components. The first term on the right hand side of the equations of motion is the pressure gradient $\left(Z_{\mathrm{s}}\right.$ is the free surface elevation), the second is the bottom friction, and the third, the horizontal diffusion. The mean bottom shear stress $\tau_{0}\left(\tau_{\mathrm{x}}, \tau_{\mathrm{y}}\right)$ enters the momentum equation (Eq. 2).

The bed (total) shear stress represents both the combined effect of skin friction and additional drag (pressure) forces acting on the bed in the presence of bedforms. In 2D models, it is related to the mean (depth-averaged) flow velocity $|U|=\sqrt{U^{2}+V^{2}}$ by a quadratic friction coefficient denoted $\mathrm{C}_{\mathrm{D}}$ :

$$
\tau_{0}=\frac{1}{2} \rho C_{D} U^{2}
$$

Different options are available for the choice of friction coefficient. Assuming a logarithmic velocity profile up to the free surface, the friction coefficient can be related to the representative equivalent bed roughness, denoted $\mathrm{k}_{\mathrm{s}}$ :

$$
C_{D}=2 \kappa^{2}\left[\operatorname{Ln}\left(\frac{30 h}{e k_{s}}\right)\right]^{-2}
$$

where $\kappa(=0.4)$ is the von Karman constant and $e=\exp (1)$.

The equivalent bed roughness coefficient $k_{s}$ is represented in terms of the characteristic bedform dimensions averaged over the grid scale. This single scale represents therefore a wide spectrum of bedforms including the effect of grain skin friction, micro-scale ripples (typically of the order of $10 \mathrm{~cm}$ ) and mega ripples and dunes that scale with the water depth. The macro-scale features (typically of the order of $100 \mathrm{~m}$ ) are typically smaller than the grid size in large scale models, and their effect needs to be parameterized also. 
The bed roughness is generally determined based on a trial and error procedure in order to reproduce the available hydrodynamic data.

The morphodynamic model Sisyphe calculates the sediment transport rates, decomposed into bed-load and suspended load, and the resulting bed evolution. The model is applicable to noncohesive sediment, composed of either uniform grains or multigrains, characterized by their mean size and density, as well as cohesive sediments and mixtures. Detailed information on the sediment transport processes can be found in the Sisyphe user manual [Villaret, 2010] and in Villaret et al. [2011].

A choice between 10 classical transport formulae is available to predict the bed load $\mathrm{Q}_{b}$ as a function of the hydrodynamic bed shear stress, corrected for skin friction. For the suspended load, we solve an additional transport equation for the depth-averaged suspended sediment concentration transport equation, where the source term represents the net erosion (E) minus deposition (D) flux.

For non-cohesive sediments, the erosion flux (E) is expressed in terms of an 'equilibrium' reference concentration [e.g. Van Rijn, 1984] which is defined at a reference elevation whereas, for cohesive sediments, the Krone-Partheniades relation relates the erosion rate to the excess bed shear stress minus the resistance of the bed to erosion, which varies as a function of mud composition and consolidation state. The deposition flux (D) is calculated as the product of settling velocity $\mathrm{W}_{\mathrm{s}}$ and the near bed concentration.

The variation of bed elevation can be derived from a simple mass balance, as expressed by the Exner equation:

$$
(1-p) \frac{\partial Z_{f}}{\partial t}+\operatorname{Div}\left(\overrightarrow{Q_{b}}\right)+(E-D)=0
$$

where $p$ is the bed porosity ( $\mathrm{p} \sim 0.4$ for non-cohesive sediment), $Z_{f}$ the bottom elevation, $Q_{b}$ the solid volume transport rate (bed-load) per unit width, and the last term represents the net erosion (E) minus deposition (D) flux.

Sediment transport rates are calculated as a function of the local skin friction, which is calculated from the hydrodynamic friction $\tau_{0}$ by use of a skin friction correction parameter $(\mu<1)$. The skin friction correction parameter $\mu$ is the ratio of skin and total friction ( $\mu=\frac{C_{d}{ }^{\prime}}{C_{d}}$ ) where $C_{d}^{\prime}$ is the quadratic friction coefficient corresponding to the skin roughness, which, for flatbed conditions, is proportional to the mean grain size $\left(k_{s}{ }^{\prime}=3 d_{50}\right)$.

\section{Internal coupling and method of feedback}

The sediment transport model (Sisyphe) relies on a complete description of the flow field, through internal coupling with the flow module; at each time step, the hydrodynamic model (Telemac-2D or -3D) calculates the flow field and sends to Sisyphe the spatial distribution of the main hydrodynamic variables: water depth $h$, horizontal depth-averaged flow velocity components $U$ and $V$, and the quadratic friction coefficient $C_{d}$. The bed roughness parameter has been identified as the key parameter regarding sediment transport rate predictions.

In order to avoid possible inconsistency between the morphodynamic model and hydrodynamic model, a method of feedback for the bed roughness, decomposed into skin friction and drag friction, has been developed [cf. Villaret et al., 2011]. According to van Rijn [2007], the total bed roughness can be decomposed into a grain roughness $k_{s}^{\prime}$, a small-scale ripple roughness $k_{r}$, a mega-ripple component $k_{m r}$, and a dune roughness $k_{d}$ :

$$
k_{s}=k_{s}^{\prime}+\sqrt{k_{r}^{2}+k_{m r}^{2}+k_{d}^{2}}
$$

Both small scale ripples $\mathrm{k}_{\mathrm{r}}$ and grain roughness $k_{s}^{\prime}$ have an influence on the sediment transport laws, while the mega-ripples and dune roughness only contribute to the hydrodynamic model (total friction). In the bed roughness feed-back method, the total bed roughness calculated by Sisyphe is sent to Telemac-2d and converted into quadratic friction coefficient. The total bed roughness is sent to the $2 \mathrm{D}$ - hydrodynamic model and converted into a friction parameter for sediment transport rate predictions, before calculating the bedload transport $\mathrm{Q}_{\mathrm{b}}$ and erosion rates $(\mathrm{E})$.

Assuming local equilibrium conditions between the flow and bed morphology, the value of the roughness for each bedform component depends on a mobility parameter $\left(\mathrm{U}^{2} / \Delta \mathrm{gd}_{50}\right.$, where $\mathrm{U}$ is the flow velocity and $\Delta$ the excess of relative density) and the median diameter of the bed material $\mathrm{d}_{50}$, with different expression for silt, sand or gravel. In summary the roughness can be expressed as a function of the following parameters (Eq. 2).

$$
k_{s}=f_{c t}\left(\frac{U^{2}}{\Delta g d_{50}}, d_{50}, d_{\text {silt }}, d_{\text {sand }}, d_{\text {gravel }}\right)
$$

where $\mathrm{d}_{\text {silt }}=0.02 \mathrm{~mm}, \mathrm{~d}_{\text {sand }}=0.062 \mathrm{~mm}$ and $\mathrm{d}_{\text {gravel }}=2 \mathrm{~mm}$. Further, the formulation of van Rijn [2007] for $\mathrm{k}_{\mathrm{s}}$ is sensitive not only to the sediment grain size but also, where dunes and mega-ripples are concerned, to the water depth.

This method has been selected as the most accurate for the lower alluvial regime (ripples and dunes) as typically met in estuarine conditions [Huybrechts et al., 2010b]. For waves and combined waves and currents, ripple dimensions can be calculated as a function of the wave parameters. Here such estimations have been implemented only in the case of the Dee estuary [see Villaret et al., 2011] again based on the methodology of van Rijn [2007] which, for combined waves and currents, utilizes a wave-current mobility number $\left(\mathrm{U}^{2}+\mathrm{U}_{\mathrm{w}}{ }^{2}\right) / \Delta \mathrm{gd}_{50}$ wherein $\mathrm{U}_{\mathrm{w}}$ is the near-bed wave velocity amplitude.

The procedure above for the prediction of the bed roughness accounts only for the turbulent form drag exerted by the physical bed roughness, i.e. the roughness associated with the physical dimensions, shapes and asymmetries of sub-grid-scale bed features, of which dunes are normally of greatest importance. In addition, however, wave-current interaction in the seabed boundary layer should, in principle, be included as a further factor enhancing the total drag felt by the (tidal) flow. Wave stirring in the near-bed layer can greatly enhance the turbulence intensity and this should be reflected, in turn, in the bed roughness $\mathrm{k}_{\mathrm{s}}$. Van Rijn [2007] proposed a formulation that typically enhances the 'physical roughness' $\mathrm{k}_{\mathrm{s}}$ by a factor of between 2 and 5 times due to wave-current interaction. One conceptual uncertainty here, however, is whether the $\mathrm{k}_{\mathrm{s}}$ enhancement should be applied to some or all of the four component parts of $\mathrm{k}_{\mathrm{s}}$ involved in Eq.(6); there is presently insufficient experimental evidence here, particularly regarding $\mathrm{k}_{\mathrm{d}}$ and $\mathrm{k}_{\mathrm{mr}}$.

Another limitation arises in the use of Eq.(6) when time-varying flows such as tidal flows are involved. While spatial variations in $\mathrm{k}_{\mathrm{s}}$ are potentially important in determining the strength of the mean flow locally in a computational domain, large temporal 
variations are typically predicted to occur also through the use of Eq.(6) instantaneously as the computation proceeds. These temporal variations in $\mathrm{k}_{\mathrm{s}}$ through a tidal cycle may, in practice, be unrealistically large, particularly for dunes, due to the present lack of any bed form history effects in our simulations. From observations made in time-varying river flows Paarlberg et al. [2010] have pointed out that observed dune heights do vary significantly in time, exhibiting a hysteresis lag with regard to the flow strength, and Coleman et al. [2010] have proposed a predictive scheme for $\mathrm{k}_{\mathrm{s}}$ in such circumstances. However, intratidal variation in $\mathrm{k}_{\mathrm{s}}$ may actually be relatively small due to the time scales required for dunes to evolve. At the present stage of this research we do not yet have sufficient field evidence to propose a suitable formulation for the history effect in $\mathrm{k}_{\mathrm{s}}$ and this represents a clear priority for future study.

\section{THE GIRONDE ESTUARY}

The Gironde macro-tidal estuary extends from the confluence of the Garonne and Dordogne Rivers to the mouth on the Atlantic coastline. Its width ranges from 3.2 to $11.3 \mathrm{~km}$ wide downstream for a length of $170 \mathrm{~km}$. The central part is characterized by a complex geometry, with the presence of different channels separated by elongated sand banks and characterized by a high turbidity level due to cohesive sediments coming from both rivers.

The estuary can be classified as macro-tidal, hyper-synchronous and with an asymmetric tide ( $4 \mathrm{~h}$ for the flood $8 \mathrm{~h} 25$ for the ebb). The tidal amplitude near the mouth of the estuary ranges from 2.2 $\mathrm{m}$ to $5.4 \mathrm{~m}$ during the spring-neap cycle, higher than $4 \mathrm{~m}$ further upstream. The river discharge ranges from 50 up to $2000 \mathrm{~m}^{3} \cdot \mathrm{s}^{-1}$. During flood events, the river flow rate becomes occasionally greater than $5000 \mathrm{~m}^{3} \cdot \mathrm{s}^{-1}$.

\section{Sediment bed composition}

The bed composition is highly variable in space: gravel and sand can be found at the mouth of the estuary as well as upstream of the tributaries. Contrarily, the channel bed is dominated by the mud except for some sand banks from the mouth to the tributaries [Allen, 1972]. Information concerning the bed material is generally provided qualitatively: areas of sand, mud or gravel are reported on maps. A measurement campaign was performed in 2009 which provides detailed quantitative information on the spatial variation of the sediment granulometry and composition in the central part: 39 samples were collected for a study area about $10 \mathrm{~km}$ length in the vicinity of the velocity measurements. The median diameter sizes from $0.01 \mathrm{~mm}$ to $0.35 \mathrm{~mm}$ and the spaceaveraged value of the median diameter for the 39 samples is 0.03 $\mathrm{mm}$. At the estuary mouth, the median diameter of the bed material ranges within 0.25 and $0.38 \mathrm{~mm}$ [Port Autonome de Bordeaux, 2002]. The bed material of the Gironde is thus composed of a mixture of mud and of highly graded sand. Some bed forms are locally observed near sand bank or at the mouth even if the mud seems to dominate the bed material in the central part. For instance, Allen [1972] reports the presence of dunes about $1 \mathrm{~m}$ height and $10 \mathrm{~m}$ long a little bit upstream the location of the velocity measurements (Fig. 1). More recently, such large scale bed forms are reported in the same area in the main channel on the right side. Typical dimensions of those small scale dunes are about $5.5 \mathrm{~m}$ in length with a height of approximately $70 \mathrm{~cm}$.

\section{Bathymetric evolutions}

In the central part of the estuary, morphodynamic features evolved drastically from 1994 to 2005 as monitored through regular bathymetric surveys (Fig. 2). Those bed evolutions are either due to anthropogenic or natural origins and may also notably be attributed to large amounts of dredging volumes.

The bed evolutions are measured through bathymetry surveys made every 5 years, since it takes about 4 years to cover the whole estuary from Bordeaux to Verdon station. A better accuracy is expected in the central part of the estuary and model validation will be focused on this part. A rather coarse grid is applied in both maritime and fluvial parts, where the bathymetry has not be updated. In the central part of the estuary, morphodynamic features evolved drastically from 1994 to 2005 and more detailed bathymetric data sets are available for years 2000 and 2005. The 1995 bathymetry is used as an initial condition of the morphodynamic model whereas the 2000 data is used to compare the measured bed evolutions with model predictions. The 2005 bathymetry is used in the hydrodynamic model validation. The 1995-2000 differential bathymetry is used for morphodynamics model validation.

\section{Hydrodynamic data}

The tide propagation can be analyzed through water levels, which are measured every $5 \mathrm{~min}$ at nine hydrometric stations along the estuary from the Verdon station at the mouth to the harbour of Bordeaux, located $10 \mathrm{~km}$ upstream of the confluence between the Dordogne and Garonne rivers (Fig. 3). Measurements of flow rates are available every hour at the upstream boundary.

Velocity measurements are sparser in comparison to the water level data. For instance, ADCP velocity profiles were measured by EDF R\&D in August 2006 at 3 points located along the same cross section, approximately $5 \mathrm{~km}$ downstream Pauillac station (Fig. 3) and at 5 points along the estuary from September to October 2009 (7 points were measured, as shown on Fig. 1, but only 5 of them were successful). Both events are used to calibrate and validate the hydrodynamic model [Huybrechts et al., 2012].

\section{Turbidity measurements}

The suspended load and related water quality parameters have been measured at four stations along the estuary [www.magest.ubordeaux1.fr] since 2005. This monitoring gives information on the turbidity variation along the estuary as a response of seasonal variation in the river flow rates. Three stations are located in the tributaries whereas one station is located in the estuary itself at Pauillac station (Fig. 3). The turbidity data can be converted into massive sediment concentration using a conversion factor of 0.0023 [Commarieu, 2007]. However during the two velocity field surveys (August 2006; September-October 2009) used to calibrate the hydrodynamics, the turbidity data are not available at Pauillac station. Therefore, two events of sediment transport data collected in August 2007 are selected: from 1 st to $5^{\text {th }}$ (spring tide) and from $7^{\text {th }}$ to $11^{\text {th }}$ (neap tide). It is assumed that the hydrodynamic conditions in August 2007 are similar as the hydrodynamic conditions in August 2006 during the velocity survey and thus that the hydrodynamic calibration of the model still remains valid. 


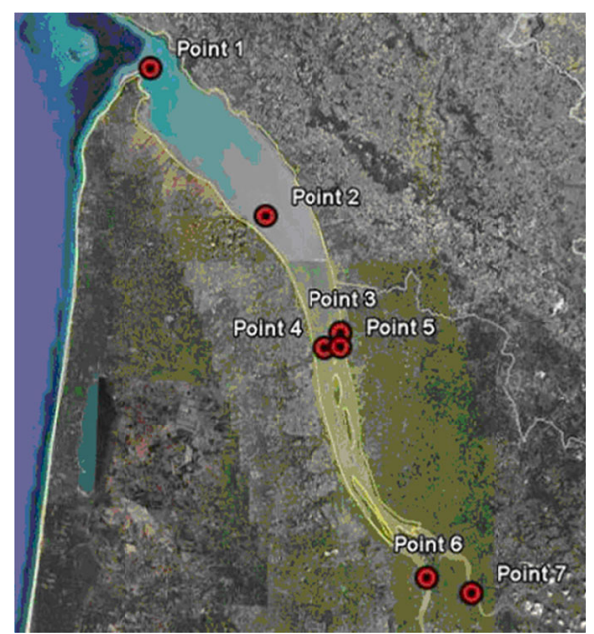

Figure 1. ADCP velocity measurements along the Gironde estuary (Sept 2009 campaign).

\section{MORPHODYNAMIC MODEL}

We present here our ongoing effort to develop a process-based model to describe the sediment transport rate and medium term evolution in the Gironde estuary. The 2D approach (Telemac$2 \mathrm{~d} /$ Sisyphe) has been selected here as a good compromise between model accuracy and CPU time.

\section{Grid mesh and extension}

The numerical domain covers the whole estuary: from the Bay of Biscay (mouth near Verdon, Fig. 3) to La Reole and Pessac, considered as the limit of the tide influence in the tributaries. The numerical domain is extended into the coastal zone $(30 \sim 40 \mathrm{~km}$ from Verdon station) in order to impose the tide height in deep water. The unstructured triangular mesh comprises 22650 nodes. The cell lengths extend from $50 \mathrm{~m}$ in the refined central part and up to $2,5 \mathrm{~km}$ offshore. At the mouth (Fig. 4(a)), a mesh size of 100 $\mathrm{m}$ is imposed in the smallest flow section. The mesh is also refined around the navigation channel where bathymetric gradients are expected. In the central part (Fig. 4(b)), the coarser mesh size is about $200 \mathrm{~m}$. The mesh becomes finer near the power plant between Trompeloup and Patiras islands.

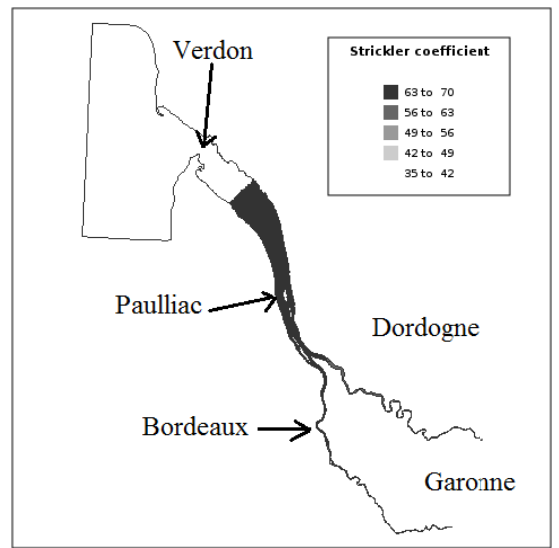

Figure 3. Spatial distribution of the calibrated friction coefficient for the hydrodynamics.

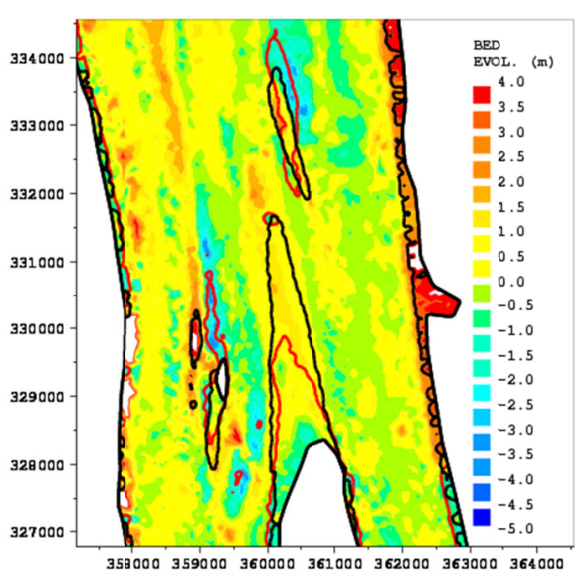

Figure 2. Bed evolution from 1995 to 2000 with iso-line zero (in red is 1995 data, in black, 2000).

\section{Boundary conditions}

The tidal components are issued from a global oceanic model [Lyard et al., 2006] and are recomposed following Schureman [1968] methodology. The tide height is composed of 46 harmonic waves [Huybrechts et al., 2011].

Flow rates are imposed at the upstream boundary and the tide height at the maritime downstream boundary for the hydrodynamics.

Equilibrium conditions are applied for the sediment transport rate (suspended sediment concentration and bed-load transport rates) in order to insure zero-evolution on the boundaries.

\section{Bed roughness prediction}

The feasibility of the van Rijn's bed roughness predictor method has been assessed, and the method of feedback leads to stable results. However in order to achieve best agreement with the measurements of flow velocity, the predicted (total) bed roughness converted into Strickler, had to be time-averaged and slightly adjusted by a trial and error procedure in order to obtain a set of best fit coefficients. The best fit (adjusted) set of friction coefficients are compared to their predicted mean values in Table 1. In order to predict the bed roughness $\mathrm{k}_{\mathrm{s}}$, information on the bed material composition (such as $\mathrm{d}_{50}$ ) needs to be provided. At the mouth and in the coastal area, a median diameter equal to 0.31 $\mathrm{mm}$ is selected. In the central part, a value $0.03 \mathrm{~mm}$ is imposed
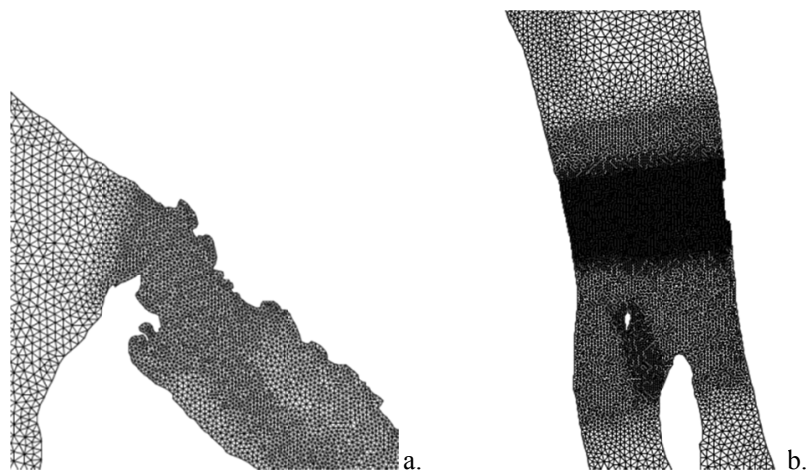

Figure 4. Grid mesh of the large scalie model (22 650 nodes); (a) Zoom of the maritime part. (b) Zoom on the central part. 


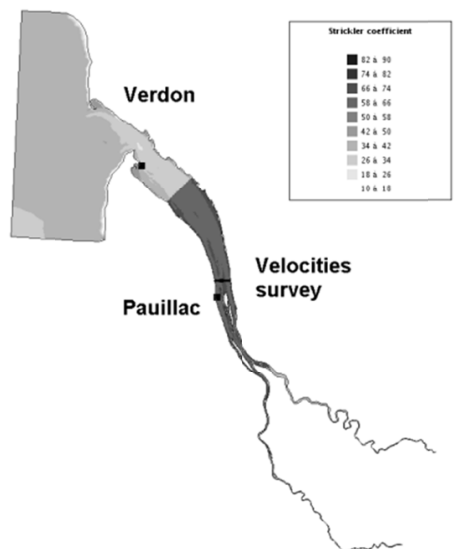

Figure 5. Spatial distribution of the calculated (Strickler) friction coefficient for the hydrodynamics based on van Rijn [2007].

[Huybrechts et al., 2010b]. At each node of the grid, a time and space variable roughness can be predicted according to the method of van Rijn. Both spatial distribution and time variation are shown on Figure 5 and 6.

At Verdon (mouth of the estuary) and Pauillac (centre part) stations, time evolutions of the predicted friction coefficient are plotted (Fig. 6). During a tidal cycle, the Strickler coefficient at Verdon varies from 28.8 to $41 \mathrm{~m}^{1 / 3} / \mathrm{s}$ with a time-averaged value of $32.6 \mathrm{~m}^{1 / 3} / \mathrm{s}$, whereas it varies from 63.6 to $66 \mathrm{~m}^{1 / 3} / \mathrm{s}$ with a timeaveraged value of $64.8 \mathrm{~m}^{1 / 3} / \mathrm{s}$ at Pauillac. The maximum Strickler coefficient corresponds to low or high tide (Fig. 6). At the mouth, the Strickler coefficient has a larger gap between the minimum and maximum value since bed forms such as dunes can develop. In the central part, the median diameter corresponds to fine cohesive material and the model predicts a quasi-constant value of the roughness which is in good agreement with the calibrated value. More significant differences are observed at the mouth where the van Rijn equation seems to predict too much friction, which is possibly due to the neglected effect of waves and wavecurrent interactions as discussed in Davies and Thorne [2008].

\section{Hydrodynamic model validation}

The hydrodynamic model results obtained with the predicted time-varying bed roughness and the calibrated constant roughness are compared with the measurements and the results previously obtained with the calibration (see Fig. 7). The top figures show the tidal signal at two points (Verdon and Pauillac stations) and the bottom figures, the velocities.

As expected, the tidal signal obtained with the van Rijn equation has a slightly smaller amplitude than the measurement at the Verdon station, which is consistent with a possible

Table 1. Comparison between predicted mean (time-averaged) and calibrated constant values of the Strickler coefficients at two stations.

\begin{tabular}{lcc}
\hline $\begin{array}{l}\text { Strickler coefficients } \\
{\left[\mathrm{m}^{1 / 3} / \mathrm{s}\right]}\end{array}$ & Verdon & Pauillac \\
\hline Predicted (mean) & 32.6 & 64.8 \\
Calibrated (constant) & 37.5 & 67.5 \\
\hline
\end{tabular}

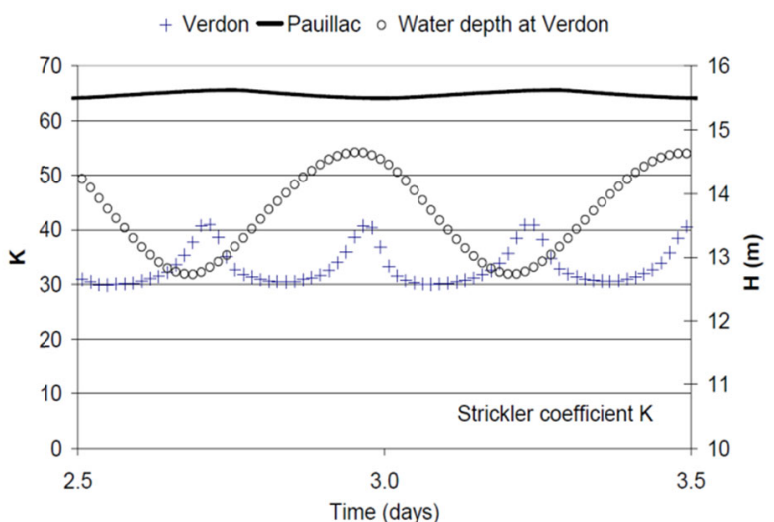

Figure 6. Time variation of the caliculated Strickler coefficient at Pauillac and Verdon stations during a tidal cycle.

overestimate of the friction in the maritime part. This may be due to the neglected effect of waves. At Pauillac, the accuracy of both model results is equivalent in comparison to the data. However, the amplitudes of the velocity signal are significantly underpredicted using the van Rijn equation. For the morphodynamic computations, the calibrated Strickler coefficients are kept, while we further assume fully non-cohesive sediments. Therefore, the effect of fine cohesive sediment which presumably inhibit the development of bedforms in the central part are implicitly included into the value of the Stickler coefficient.

\section{Morphodynamic model results}

In the present contribution, the bed material is treated as a mixture of silt, fine sand and medium sand. For this test case, the cohesive effects are not included into the sediment transport. Some new developments on the cohesive effects are still in process [Van et al., 2011] to integrate for instance mud consolidation.

The variability of the sediment distribution along the Gironde estuary is schematized by assuming an initial uniform sediment distribution for each geo-morphological unit. In the upper river part, the bed sediment is composed of silt $\left(\mathrm{d}_{50}=60 \mu \mathrm{m}\right)$, whereas the maritime part is made of medium sand $\left(\mathrm{d}_{50}=310 \mu \mathrm{m}\right)$. In the central part, the bed is made of a mixture of $50 \%$ of fine sand $\left(\mathrm{d}_{50}=210 \mu \mathrm{m}\right)$ and $50 \%$ of silt $\left(\mathrm{d}_{50}=60 \mu \mathrm{m}\right)$.
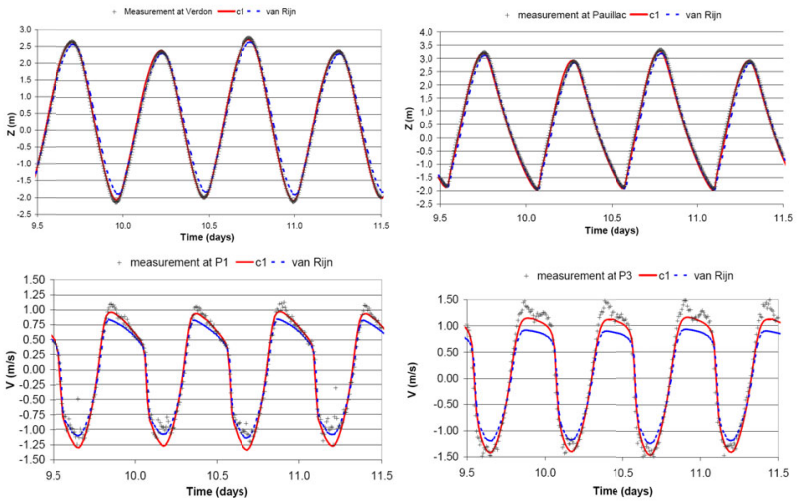

Figure 7. Comparison between the model results (c1 in red are the calibrated results $c 2$, in blue the results obtained with the van Rijn predictor). The black points are the data. 


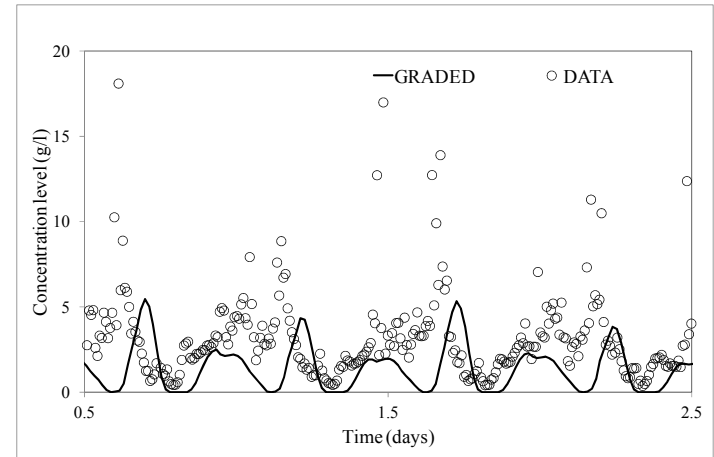

Figure 8. Time-varying concentration in $\mathrm{g} / \mathrm{l}$ calculated at Pauillac ( $t=0$ corresponds to the August $1^{\text {st }} 2007$ (OhTU).

The grain size distribution calculated by the model after one year is shown on Fig. 9(b). In the maritime part, the fine sediment is flushed out and deposited offshore, which is in qualitative agreement with observations (Fig. 9(a)). In the central part, very fine (cohesive) sediment is dominant downstream of the Patiras island and deposits area, whereas coarser sediment is predominant in the deeper channels, as observed by Boucher [2009].

Sediment transport predictions are highly sensitive to the sediment granulometry and bed composition as well as to the choice of transport formula. In the present application, transport rates are dominated by the presence of very fine particles in suspension. The suspended load is highly sensitive to the choice of settling velocity, which can be deduced from the grain diameter using a semi-empirical formula [cf. van Rijn, 1984]. The reference length delineating the bed-load and suspended load is taken at 0.5 $\mathrm{k}_{\mathrm{s}}$ as suggested by van Rijn, where $\mathrm{k}_{\mathrm{s}}$ is the equivalent bed roughness. Influences of the ripples on the skin friction and thus on the transport rates are incorporated for all the performed computations. Model results are in qualitative agreement with turbidity measurements from the Magest survey. Time-varying
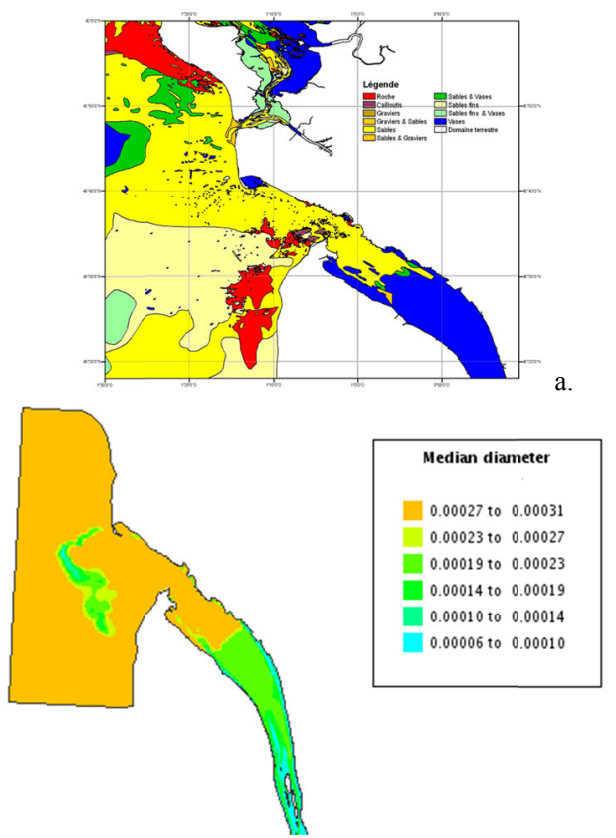

b.

Figure 9. Grain size distribution: (a) Measurements (provided by Shom). (b) Predicted mean diameter. concentrations calculated at the Pauillac station are shown in Figure 8 .

Best agreement in the central part, at Pauillac station, is obtained by adjusting the settling velocity to $1.8 \mathrm{~mm} / \mathrm{s}$ for the finer grain size and by lowering the empirical coefficient in the van Rijn formula ( 0.05 instead of 0.15$)$. In comparison to the data (see Fig. 7), the model tends globally to underestimate the peaks in concentrations. The overall time-variation is qualitatively well reproduced.

In the large scale morphodynamic model, the sequence of dry or flood seasons can be imposed at the upstream boundaries based on measured flow rates from January $1^{\text {st }} 1995$ to December $31^{\text {st }} 2000$. During this sequence of dry and flood seasons, the flow rate in the Garonne River decreases down to $60 \mathrm{~m}^{3} / \mathrm{s}$ during the dry season and reaches its maximum, up to $4000 \mathrm{~m}^{3} / \mathrm{s}$, during winter floods. In Fig. 10(b), we show the 5-year bed evolution. The bed evolution is overall, in both qualitative and quantitative agreement with the 5-year differential bathymetry, shown in Fig. 10(a).

The growth rate of the Patiras island and associated deposition rates of the fine particles downstream of the island are overestimated by roughly a factor 2 . This discrepancy may be due to some uncertainty in the representation of physical processes as well as some uncertainty in the bathymetric data. Indeed, the 1995 historical bathymetry data set is based on digitalized bathymetrical maps, from mono-beam bathymetry (the original data was not available). So the final uncertainty in the differential bathymetry is difficult to assess. The measured bed evolution in the vicinity of the banks is probably unrealistic.

The model gives overall reasonable estimates of the bed evolution despite the fact that the calculated shape of the deposit in the lee of the central island is nott well reproduced. This is not surprising since this bank is dominated by mud processes which are not presently accounted for.
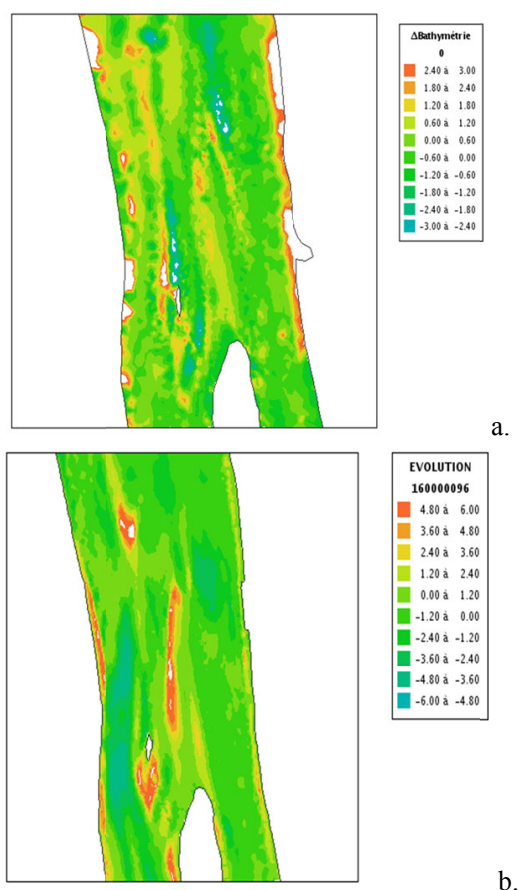

Figure 10. 5-year Bed evolutiom in the central part (19952000): (a) The differential bathymetry. (b) The calculated bed evolution. 


\section{CONCLUSIONS}

A 2D morphodynamic process-based model has been applied to predict the bed evolution in a complex estuarine system: the Gironde estuary. Thanks to recent progress in the numerical methods and extensive use of parallelization, medium term predictions (order of decades) can be achieved at the basin scale $(150 \mathrm{~km})$ without the use of hydrodynamic filtering methods nor morphodynamic factors. With a time step of $100 \mathrm{~s}, 5$ years of bed evolution requires approximately $18 \mathrm{hrs}$ of computational time using 8 processors (Intel Xeon-3.33 GHz).

The model (Telemac-2d/Sisyphe) presented here takes into account complex interacting processes between the flow and sediment transport. In particular, a method of feedback for the bed roughness which avoids inconsistencies between the flow and sediment transport model, has been proved to give physically realistic as well as stable numerical results. For validation, model results could be further improved by slightly adjusting the predicted time-averaged mean friction coefficients in order to get a set of best fit coefficients (constant). The bed friction predictor can therefore be viewed as a way to guide a time-consuming trial and error procedure, on physically sound basis.

Sediment transport processes include the coupling between bedload and suspended load, as well as sand grading effects. Here only 3 sand classes were considered which is a very crude schematization of the high heterogeneity in the sediment bed composition. We only account for the non-cohesive processes. The effect of cohesion and mixed sediment processes needs to be accounted for using the consolidation model [cf. Van et al., 2011]. The effect of waves is discussed in Villaret et al. [2011].

\section{ACKNOWLEDGEMENTS}

The authors thank the "Grand Port Maritime de Bordeaux (GPMB)", the "Service Hydrographique et Océanographique de la Marine (SHOM)", Etablissement Public Territorial du Bassin de la Dordogne" and "Département de l'équipement- DDE 33 for providing their data.

\section{REFERENCES}

Allen G.P., 1972, Etude des processus sédimentaires dans l'estuaires de la Gironde, Phd Thesis Université de Bordeaux. $314 \mathrm{p}$.

Boucher, O., 2009, Note sur les analyses de granulométrie Laser sur les échantillons de sédiments prélevés en Gironde, rapport Cetmef.

Chini N., Villaret C., 2007: Numerical modelling of the bed evolution downstream of a dike in the Gironde estuary, Proceedings of the River, Coastal and Estuarine Morphodynamics Conference. $7 p$.

Coleman, S. E., Zhang, M. H., \& Clunie, T. M. (2005). Sediment-Wave development in subcritical water flow. Journal of Hydraulic Engineering, 131(2), 106-111.

Commarieu M.V., 2007: Oxygénation des eaux dans un estuaire hyperturbide, observations in-situ, expérimentation et modélisation, Thèse de l'Université de Bordeaux, $175 \mathrm{p}$.

Davies, A.G. , Thorne P.D., 2008: Advances in te study of moving sediments and evolving seabed, Surv. Geopys, DOI.1007/s10712-008-9039-x
Hervouet J.M., 2007: Hydrodynamics of free surface flow modelling with the finite element method, Wiley. ISBN 978-0470-03558-0, 341p.

Hervouet J.M., Razafindrakoto E., Villaret C., 2011: Dealing with dry zones in the free surface flow, a new class of advection schemes, IAHR Congres. Brisbane, Australia.

Huybrechts N., Villaret C., Lyard F., 2011: Optimized predictive 2D hydrodynamic model of the Gironde estuary (France). (...Journal of Waterway, Coast, Port and Ocean engineering).

Karunarathna H., Reeve D., Spivak M., 2008: Long term morphodynamic evolution of estuaries: an inverse problem, Estaurine Coastal and Continental Shelf Science 77, 385-395.

Lesser G.R., Roelvink J.A., van Kester J.A.T.M., Stelling G.S, 2004: Development and validation of a three dimensional morphological model, Coastal Engineering, 51, pp. 883-915.

Lyard, F., Lefevre, F., Letellier, T., Francis, O., 2006: Modelling the global ocean tides: modern insights from FES2004", Ocean Dynamic, Vol 56 (5-6), pp 394-415.

Paarlberg, A.J., Dohmen-Janssen, C., Hulscher, J.M.H., Termes, P., \& Schielen, R. (2010). Modelling the effect of timedependent river dune evolution on bed roughness and stage. Earth Surface Processes and Landforms, 35, 1854-1866 (2010) DOI: 10.1002/esp.2074.

Papanicolaou A.N., Elhakeel M., Krallis G., Prakash S., Edinger J., 2008 : Sediment transport modelling review - Current and Future Development, Journal of Hydraulic Engineering, 134(1).

Schureman, P. (1958) "Manual of harmonic analysis and prediction of tides", US Department of Commerce, Coast and Geodetic Survey, Washington, US.p317.

Van L.A; Pham Van Bang D., 2011: On the self weight of consolidation mixtures, River Coastal Estuarine Morphodynamics Conference, Beijing sept. 2011.

Van der Wegen, M., Roelvink, J.A., 2008: Long term morphodynamic evolution of a tidal embayment using a 2D process-based model, Journal of Geophysical Research, Vol. 113, C03016, doi: 10.10292006JC003983.

Van der Wegen, M. 2010, Modeling morphodynmaic evolution in alluvial estuaries, PHD Thesis, Delft University of Technology, ISBN 978-0-415-59274-1 (Taylor \&Francis Group).

Van Rijn, L.C. 1984 : Sediment transport, part III: bed forms and alluvial roughness, J. Hydraul. Eng.-Asce, 110(12), 1733-1754.

Van Rijn, L.C., 2007: Unified view of sediment transport by currents and waves. 1. Initiation of motion, bed roughness, and bed-load transport", J. Hydraulic Eng. 133( 6), pp 649-667.

Villaret, C., et al., 2011: Morphodynamic modeling using the Telemac finite-element system. Computers \& Geosciences, doi:10.1016/j.cageo.2011.10.004

Villaret C., Huybrechts N., Davies A.G., Way O., 2011: Effect of bed roughness prediction on morphodynamic modelling: Application to the Dee estuary (UK) and to the Gironde estuary (France), Proceedings of the $34^{\text {th }}$ IAHR World Congress, Brisbane (Australia).

Warner J.C., Sherwood C.R., Signell R.P., Harris C.K., Arango H.G., 2008: Development of a three-dimensional, regional, coupled wave current and sediment transport model, Computer and Geosciences, 34, pp. 1284-1306.

Warren, I.R., Bach, H.K., 1992. MIKE 21: a modelling system for estuaries, coastal waters and seas. Environmental Software 7 (4), $229 \mathrm{e} 240$. 